\title{
Star Formation History of the Dwarf Galaxies in the Centaurus A Group
}

\section{Makarova ${ }^{1}$, D. Makarov ${ }^{1}$, A. Dolphin ${ }^{2}$, I. Karachentsev ${ }^{1}$, B. Tully ${ }^{3}$,} S. Sakai ${ }^{4}$, E. Shaya ${ }^{5}$, L. Rizzi ${ }^{3}$, M. Sharina ${ }^{1}$, and V. Karachentseva ${ }^{6}$

${ }^{1}$ Special Astrophysical Observatory, Russia; ${ }^{2}$ Steward Observatory, USA; ${ }^{3}$ IfA, Univ. of Hawaii, USA; ${ }^{4}$ Univ. Of California, Los Angeles, USA; ${ }^{5}$ Univ of Maryland, USA;

${ }^{6}$ Astronomical Observatory of Kiev, Ukraine

We derive quantitative star formation histories ( $\mathrm{SFH}$ ) of the two dwarf spheroidal (KK 197 and ESO 269-066) and one dwarf irregular (ESO 381-018) galaxies in the nearby Centaurus A group. The data are part of our sample of about 50 nearby dwarf galaxies observed with the Advanced Camera for Surveys (ACS) at the Hubble Space Telescope (prog. 9771 \& 10235, PI I.Karachentsev). Deep color-magnitude diagram (CMD) of KK 197 is shown in Fig.1.

Photometry of resolved stars in the galaxies was made by A.Dolphin with his DOLPHOT package for crowded field photometry. Photometric distances for all galaxies in the sample were obtained using tip of the red giant branch (TRGB) distance indicator (Lee et al. 1993, ApJ, 417, 553; Sakai et al. 1996, ApJ, 461, 713). We also used our StarProbe package for quantitative SFH determination (Makarov \& Makarova 2004, Ap, 47, 229).

The CMDs of ESO 269-066 and KK 197 are typical for dSphs which mostly populated with old RGB stars. The diagrams are contaminated by a number of foreground stars. However, we should also suppose a presence of intermediate age AGB stars. These galaxies have unusual RGB color scatter, which reflect active star formation episode of very high metallicity level (Fig.2). The probable explanation can be that the galaxies have captured the material lost by galaxy in merging with Centaurus A. At the same time the SFH of more distant ESO 381-018 is quite typical for dIrr galaxies. It has not metal rich stars or very high level of star formation.

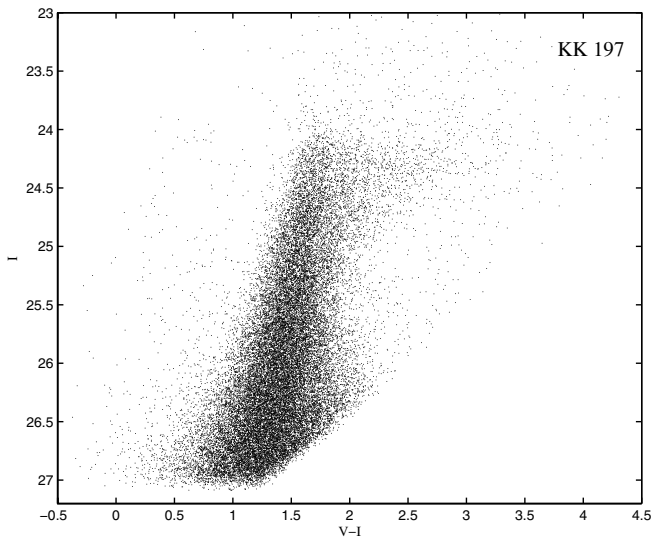

Fig.1. I,V-I CMD of KK 197. The derived distance is $3.87 \mathrm{Mpc}$ (Karachentsev et al. 2006, accepted in AJ).

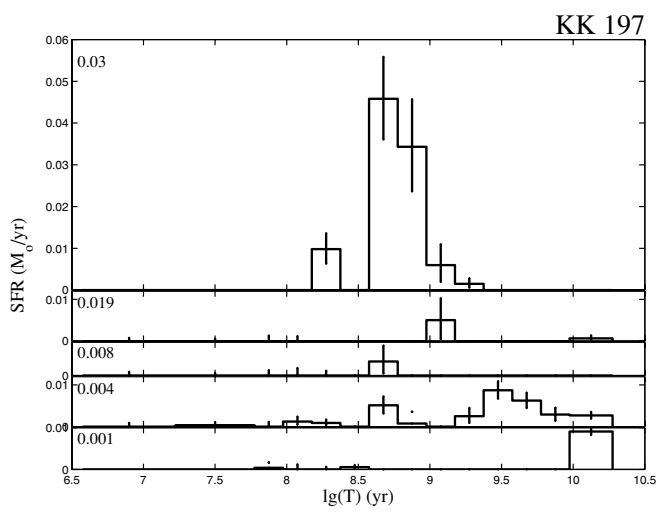

Fig.2. Star Formation History of KK 197. 